\title{
Propuesta de prototipo de un sistema experto para el diagnóstico mecánico y eléctrico de automóviles
}

\section{Proposal for a prototype of an expert system for the mechanical and electrical diagnosis of automobiles}

\begin{abstract}
Alexandra Agudo ${ }^{l}$, Aarón Delgado ${ }^{l}$, Víctor Carrión ${ }^{l}$, Cristian Pinzón ${ }^{2 *}$
${ }^{1}$ Licenciatura en Ingeniería de Sistemas y Computación, Centro Regional de Veraguas, Universidad Tecnológicas de Panamá,

${ }^{2}$ Facultad de Ingeniería en Sistemas Computacionales, Centro Regional de Veraguas, Universidad Tecnológicas de Panamá

Resumen El vehículo es un recurso indispensable en la sociedad actual para llevar a cabo las actividades laborales, sociales, de ocio, emergencias, etc., donde su mantenimiento es fundamental para su funcionamiento correcto. Sin embargo, en muchas situaciones nos encontramos imprevistos con el automóvil que pueden ser evitados si se pueden detectar anomalías a tiempo, o en cualquier caso tener un diagnóstico preliminar del problema del automóvil, antes de recurrir a un especialista y evitar grandes costos de reparación. Este artículo presenta la propuesta de un prototipo de un sistema experto para diagnosticar fallas mecánicas o eléctricas comunes en un automóvil. Este prototipo está pensado para los usuarios que generalmente desconocen acerca del funcionamiento o mantenimiento que necesite su automóvil. El trabajo se basó en una investigación descriptiva identificando un problema y planteando una solución al problema identificado. Para la construcción del prototipo se evaluaron diferentes herramientas que se ajustaran al problema, en ese sentido la herramienta VisiRule fue la seleccionada. VisiRule presenta ventajas con respectos a otras herramientas tales como su facilidad para representar el problema y estrategia acelerada para la construcción de prototipos. En esta primera etapa del proyecto, se generó un primer prototipo del sistema experto que abarca los problemas generales y comunes sobre fallas electromecánicas en un automóvil. En este trabajo se describe de manera detallada la construcción del prototipo, la metodología aplicada, los resultados y conclusiones obtenidas.
\end{abstract}

Palabras clave Automóviles, diagnóstico, inteligencia artificial, mecánica, sistema experto.

\begin{abstract}
The vehicle is an indispensable resource in today's society to carry out work, social, leisure, emergency activities, etc., where its maintenance is essential for its correct operation. However, in many situations we find unforeseen events with the car that can be avoided if anomalies can be detected in time, or in any case have a preliminary diagnosis of the car problem, before resorting to a specialist and avoiding large repair costs. This article presents the proposal for a prototype of an expert system to diagnose common mechanical or electrical faults in a car. This prototype is intended for users who generally do not know about the operation or maintenance that their car needs. The work was based on a descriptive investigation identifying a problem and proposing a solution to the identified problem. For the construction of the prototype, different tools that fit the problem were evaluated, in that sense the VisiRule tool was selected. VisiRule has advantages over other tools such as its ease of representing the problem and its accelerated strategy for prototyping. In this first stage of the project, a first prototype of the expert system was generated that covers general and common problems about electromechanical failures in a car. In this work, the construction of the prototype, the applied methodology, the results and the conclusions obtained are described in detail.
\end{abstract}

Keywords Car, diagnosis, artificial intelligence, mechanics, expert system.

* Corresponding author: cristian.pinzon@utp.ac.pa

\section{Introducción}

Actualmente no existe una herramienta de software basada en un sistema experto, para un tipo de usuario común, que le brinde recomendaciones y diagnósticos sobre el estado mecánico y eléctrico de un automóvil. Además, muchas personas, propietarios de automóviles no tienen el conocimiento del funcionamiento o mantenimiento que requiere su auto. Sobre esta base se ha propuesto un prototipo de sistema experto que pueda informar al usuario sobre el estado del vehículo ante un fallo mecánico o eléctrico.

Un sistema experto para el diagnóstico del estado de un automóvil puede ser una herramienta muy beneficiosa para el usuario en general, pero dependerá de la calidad de la propia herramienta para realizar un diagnóstico certero y recomendar una solución clara y precisa. El sistema debe contar con una 
interfaz sencilla y amigable para facilitar el uso de la herramienta y con una base de conocimiento amplia y un motor de inferencia validado.

Una persona, propietaria de un vehículo, al no contar con una herramienta que los oriente, como lo hiciera un profesional del área ante un fallo del vehículo, suelen pedir consejos de manera desesperada a amigos, familiares y otras personas de confianza. En ocasiones esto puede resultar poco eficaz para determinar el estado real del automóvil y podría acarrear un problema más serio en los componentes, y en el peor de los escenarios caer en manos no especialistas, o provocar un accidente que lamentar.

No todas las personas tienen conocimientos básicos en mecánica, que le permitan determinar de manera inmediata como se encuentra nuestro automóvil. Por ello, proponemos un prototipo para el diagnóstico de fallas mecánicas y eléctricas de un automóvil para apoyar en la toma de decisión de la persona y acelerar la solución al problema mecánico del vehículo, o en el mejor de los casos, prevenir un daño mayor o un accidente detectando de forma temprana algún tipo de falla.

El documento ha sido estructurado de la siguiente manera. La sección 1 plantea una introducción. En la sección 2 se presentan los antecedentes del trabajo. Posteriormente se presenta la metodología y los conceptos afines al tema. Seguido, se presenta el diseño y construcción del sistema propuesto. Finalmente, se presentan los resultados y conclusiones.

\section{Antecedentes}

Pocas herramientas, dirigidas a los usuarios, se han desarrollado como apoyo para detectar fallo en los vehículos, que no requieran un conocimiento básico en mecánica. A nivel profesional, los talleres de mecánica si utilizan dispositivos sofisticados, tipo escáner, como se aprecia en la figura 1, que trabajan sobre una base de código de fallas en las cuales se muestran las averías que pueda tener el auto. Sin embargo, llevar el vehículo a un taller para obtener el código de falla o el diagnóstico puede resultar costoso para los usuarios, cuando posiblemente el fallo puede ser algo sencillo de resolver.

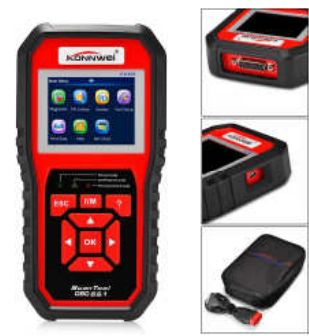

Figura 1. Ejemplo de un escáner automotriz.

Fuente: konnwei.com

Desde que se tiene el ideal de poder computarizar el conocimiento humano para ayudar en la toma de decisiones buscando poder ahorrar tiempo en dar solución a un problema, se da el surgimiento de los sistemas expertos [1]. En esta dirección se plantea este proyecto, una herramienta de software que esté disponible para cualquier usuario para tener un diagnóstico preliminar sobre alguna falla en su vehículo, y sobre esa base tomar decisiones.

\subsection{Proyectos similares}

A continuación, se listan algunas aplicaciones desde el punto de vista de los sistemas expertos en la solución de problemas reales en el área de motores.

- DELTA (Diesel Electric Locomotive Troubleshooting Aid)

Sistema experto para el diagnóstico y reparación de fallas en locomotoras diésel-eléctricas. Este sistema también es considerado uno de los pioneros en la detección de fallas en motores, se desarrolló de 1981 a 1984 [2].

- Sistema Experto STEAMER

Creado para brindar ayuda a la armada de los Estados Unidos sobre plantas de propulsión a vapor como las que son utilizadas en los buques de propulsión de vapor. Este sistema fue desarrollado por Navy Research Perssoner Development Center [2].

- Proyecto Eolo CN-235. Este sistema se basa en la enseñanza de pilotos y técnicos de mantenimiento de aviones del modelo CN- 235 [2].

- Análisis y clasificación de sonidos de motores de carros, para diagnosticar fallas, con ayuda de redes neuronales. Este sistema se encarga de clasificar y detectar las posibles fallas que puede tener el motor de un auto, utilizando únicamente el sonido grabado de la máquina [3].

- Diagnóstico de fallas de un motor de encendido provocado Corsa Evolution 1.4L mediante el análisis de las señales de los sensores MAP, TPS, VSS, utilizando herramientas de aprendizaje y clasificación. Consiste en detectar fallos presentes en el motor de combustión interna, utilizando herramientas de clasificación y aprendizaje para disminuir costos de mantenimiento [4].

- Sistema de diagnóstico de fallas en la dirección asistida eléctrica de automóviles. Creado para diagnosticar las fallas más relevantes de este sistema de dirección asistida en los autos. En una primera etapa está basado en la generación de redundancia analítica para que detecte la falla y en una segunda etapa informe el diagnóstico de la falla detectada [5].

Dentro de los proyectos similares, también podemos mencionar un sistema basado en redes neuronales para la detección de fallas [6]; y también un sistema experto la gestión de mantenimiento[7].

Como se puede ver, existen iniciativas en la utilización de sistemas expertos para el diagnóstico de fallas en componentes 
mecánicos y eléctricos, que son utilizados principalmente por profesionales, o en proyectos complejos. Sin embargo, el proyecto propuesto se planea para usuarios o dueños de vehículos, con poco conocimiento en el área de mecánica.

\section{Metodología}

En todo proyecto de investigación es importante plantear la metodología de la investigación utilizada. Este proyecto se plantea como una investigación aplicada, dado que se enfoca a la solución de un problema real. Tomando en cuenta el alcance de la investigación, se considera como una investigación descriptiva, ya que describe el problema y la solución encontrada.

En esta sección mostraremos la metodología de investigación que hemos utilizado para la propuesta de este proyecto.

\subsection{Identificación del problema}

Se realizó un análisis de la situación actual en cuanto a sistemas de ayuda y mantenimiento electromecánico. Se conversó con algún especialista en el área de mecánica para indagar sobre los problemas más básicos en un vehículo. Se pudo conocer que una gran cantidad de personas, son dueño de vehículos, pero no tienen ningún tipo de conocimiento sobre fallas de vehículos, o desconocen los recursos que el vehículo dispone para avisar de fallas. Según el especialista entrevistado, en los talleres de mecánica el cliente debe esperar en muchas ocasiones mucho tiempo para conocer el estado de su auto y luego que puedan reparar o darle el manteamiento adecuado. Además, en algunas ocasiones, el problema puede ser tan sencillo como cambiar un fusible de la caja de control, y que puede ser resuelto por el dueño del vehículo.

\subsection{Revisión bibliográfica}

Se realizó una búsqueda de información en fuentes bibliográficas sobre herramientas informáticas para diagnosticar fallas en vehículos. Igualmente, se consultó sobre las fallas más comunes en los vehículos para plantear una estrategia de solución desde el enfoque de los sistemas expertos.

En cuanto a la selección para construir el prototipo del sistema experto, se planteó una comparativa de herramientas proporcionadas. A través de este proceso, pudimos encontrar la herramienta más adecuada para la realización de un prototipo de sistema experto para el diagnóstico mecánico y eléctrico de automóviles.

\subsection{Recolección de datos}

Se realizó una revisión profunda de datos e información ya existentes en documentos sobre la mecánica automotriz y sistemas expertos. A través de la búsqueda de fuentes bibliográficas se logró obtener manuales de detección de fallas mecánicas y las soluciones aplicables a distintos casos, además de contar con el apoyo de un especialista en la mecánica automotriz.

\subsection{Selección de tecnologías}

Para el diseño del sistema, primero se crea una base de conocimiento recolectada a partir de una revisión bibliográfica. Posteriormente, se compara y se prueban herramientas comerciales para la creación de sistemas expertos. Finalmente se genera un árbol de decisiones para la creación del sistema experto, con la herramienta seleccionada.

\subsection{Diseño de la propuesta}

Se diseñó un esquema conceptual que contiene todas las heurísticas o reglas que hemos planteado para introducir en la base de conocimiento.

\subsection{Resultado}

Como resultado, se tiene establecido la creación de un prototipo de sistema experto para el diagnóstico mecánico y eléctrico de automóviles, que sea amigable y funcional para los usuarios que no tengan los conocimientos básicos sobre detección de fallas mecánicas.

\section{Conceptos}

\subsection{Inteligencia artificial}

La Inteligencia Artificial (IA) son un conjunto de algoritmos creados para emular comportamientos de razonamiento humano. En este proyecto se utiliza el concepto de IA, concretamente la utilización de los sistema expertos [8].

\subsection{Sistemas expertos}

Los sistemas expertos están basados en el conocimiento en un área específica. Son sistemas que no están capacitados para aprender por sí solos. Requieren de un conjunto de hechos y reglas previamente programadas [9].

Existen diversos tipos de sistemas expertos como lo pueden ser los basados en reglas que son previamente establecidas, los basados en casos, redes bayesianas y sistemas expertos difusos.

En este proyecto se plantea el diseño de un prototipo de sistema experto basado en reglas para realizar un diagnóstico eléctrico y mecánico en automóviles para así detectar posibles fallas y recomendar soluciones.

\subsection{Estructura de los sistemas expertos}

Los sistemas expertos están compuestos de dos partes que son [10]:

- Ambiente de desarrollo: es utilizado para introducir el conocimiento en la base de conocimiento del sistema experto.

- Ambiente de consulta: esta parte es la utilizada por los usuarios que no son expertos en un tema o área de consulta. 


\subsection{Componentes de un sistema experto}

Un sistema experto cuenta con varios componentes esenciales como se plante en [10]:

- La base de conocimiento: es la que cuenta con todo el conocimiento necesario para comprender, formular y resolver problemas. Es decir, son las heurísticas o reglas que dirigen el conocimiento para resolver el problema y responder a la consulta del usuario.

- Base de hechos: esta base de hechos alberga los datos de los problemas que el usuario desea resolver.

- Motor de inferencia: el motor es el cerebro del sistema experto. Se encarga de interpretar las reglas que se encuentran en la base de conocimiento del sistema experto. Este componente es muy esencial para el programa.

\subsection{Diagnóstico de un sistema experto}

El diagnóstico es el resultado obtenido por el motor de inferencia [10]. El concepto de diagnóstico se utiliza en nuestro proyecto para referirse al estado actual de un automóvil para detectar sus posibles fallas y encontrar una solución.

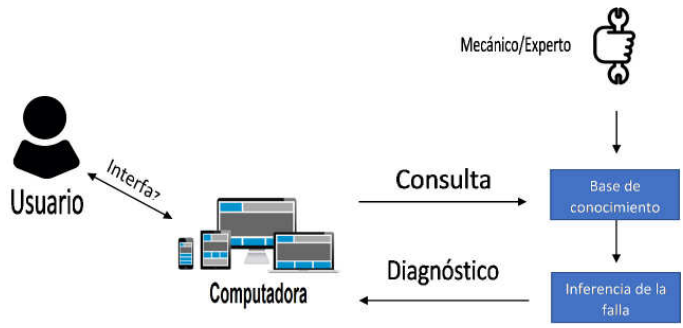

Figura 2: Diseño conceptual del sistema experto.

\section{Diseño}

Para la confección del prototipo se ha planteado el diseño conceptual para el sistema experto en la figura 2.

El usuario interactuará por medio de la interfaz del sistema experto. El sistema experto será compatible con cualquier computador disponible por el usuario.

La consulta realizada por el usuario se traslada al motor de inferencia y la base de conocimiento del sistema experto. Esta base de conocimiento fue construida a partir de una profunda búsqueda bibliográfica en temas de la especialidad y con el apoyo de un especialista en mecánica. Una vez se recibe la consulta, el sistema experto infiere una respuesta y genera el diagnóstico del vehículo para que luego el usuario pueda tomar una decisión.

\subsection{Herramienta para la construcción del sistema experto}

Para la construcción de este prototipo utilizaremos la herramienta comercial VisiRule [11]. En la figura 3 se puede apreciar el entorno gráfico de la herramienta.

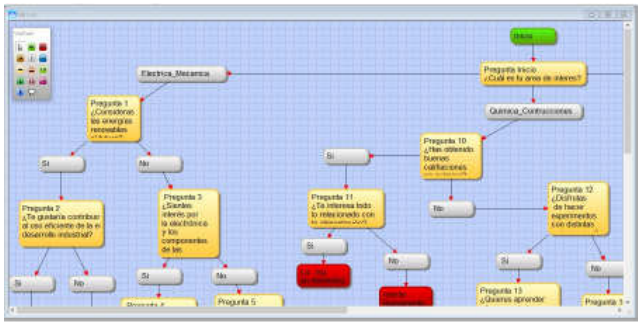

Figura 3: Interfaz de la herramienta VisiRule [11].

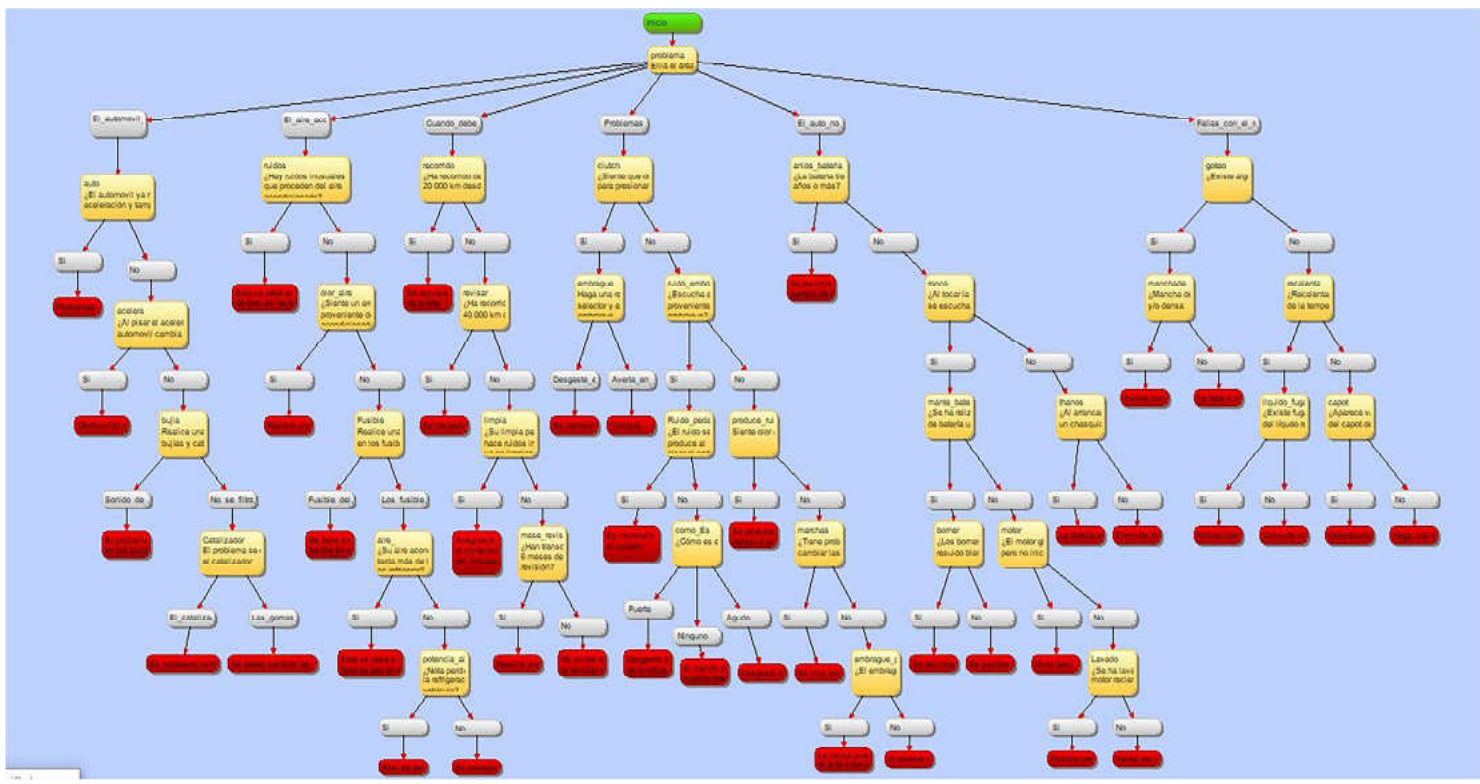

Figura 4. Interfaz de la herramienta VisiRule con el árbol de decisión. 
VisiRule es una herramienta gráfica para diseñar, desarrollar y entregar aplicaciones de soporte de decisiones y reglas comerciales [12]. El usuario puede dibujar un diagrama de flujo que represente la lógica de decisión. Los componentes principales de esta herramienta están constituidos por varios bloques predefinidos, y los componentes cruciales son cajas de preguntas con varias posibilidades de respuesta definitorias. El sistema opera bajo Windows en el entorno WIN-Prolog.

En la figura 4 se puede apreciar el árbol de decisión construido con la herramienta VisiRule que dará soporte al sistema experto para el diagnóstico de fallas en vehículos. El árbol de decisión abarca las fallas más comunes que podemos encontrar a nivel mecánico o eléctrico en los vehículos.

\section{2 Árbol de decisión del sistema experto}

En una primera etapa de diseño, se genera un árbol de decisión en la herramienta Cmaptools [13] para evaluar las opciones de diagnóstico planteadas. Se ha creado un árbol de decisión que contiene un conjunto de reglas para luego construir el prototipo del sistema experto en la herramienta VisiRule.

Los árboles de decisiones son un método de representación del conocimiento de forma gráfica, que permite entender cómo se infiere una respuesta. La estructura del árbol de decisión parte de un nodo raíz, conjunto de ramas y nodos, y los nodos terminales o conclusión. Esta estructura fue utilizada en el proyecto en la etapa de diseño del sistema.

\section{Resultados}

Se ha podido cumplir con el objetivo que fue planteado al inicio del proyecto, construir un prototipo de un sistema experto para el diagnóstico de fallas mecánicas y eléctricas en vehículos. En los siguientes subpuntos se explican en más detalle con los resultados obtenidos de las pruebas del prototipo.

\section{1 Árbol de decisión en la herramienta}

Se logró obtener una cantidad de 40 soluciones posibles para este prototipo de sistema experto. Como se observa en la figura 4, se presenta una vista general del árbol de decisiones en la herramienta VisiRule.

Luego de generar un árbol en la herramienta Cmaptools en la etapa de diseño, se realiza la implementación en VisiRule para la construcción del prototipo. Este árbol contiene cada una de las reglas que usará el sistema.

\subsection{Ejecución del prototipo}

Como resultado final de la ejecución del prototipo, presentados en las figuras 5, se ha puesto a prueba el prototipo demostrando su funcionalidad. Es importante aclarar que por ahora el prototipo es funcional solo a nivel de una computadora por las restricciones de la herramienta VisiRule.
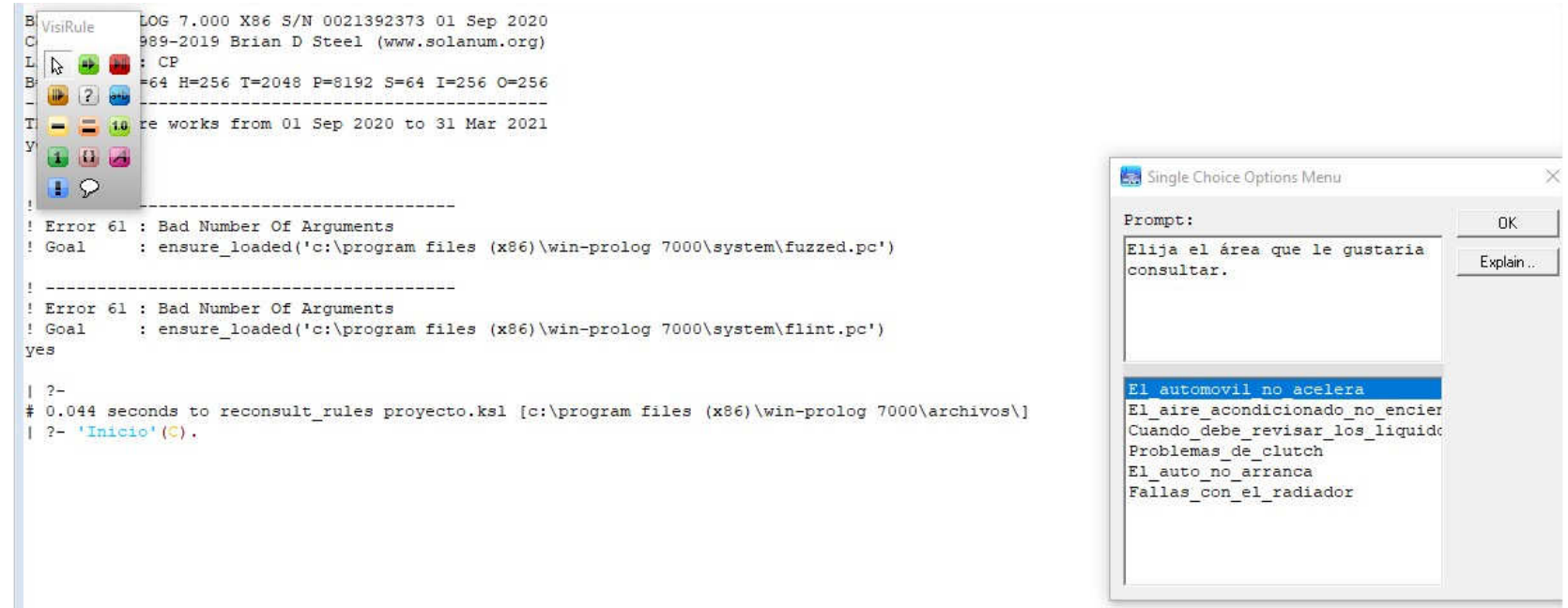

Figura 5: Ejecución por consola del sistema.

Para la realización de pruebas del prototipo, se han ejecutado por medio de la consola de la herramienta VisiRule, generando en modo texto la conclusión del prototipo.

Para evaluar el funcionamiento del sistema experto, se han realizado diversas pruebas para identificar las limitaciones. A continuación, se muestra en la tabla 1 los resultados de las pruebas.

Como se puede ver en la tabla 1, el prototipo puede cubrir los problemas electromecánicos más generales y comunes en automóviles; sin embargo, esto no representa una versión final, 
ya que se pretende mejorar el diagnóstico incluyendo fallas más complejas, para así lograr una total eficacia en el sistema. También hacer compatible el sistema con el protocolo OBD2, para futuras mejoras.

\begin{tabular}{|c|c|c|c|}
\hline Prueba & Caso & $\begin{array}{r}\text { Respuesta } \\
\text { del Sistema }\end{array}$ & Resultado \\
\hline 1 & $\begin{array}{l}\text { El auto no } \\
\text { arranca, hay } \\
\text { ruidos } \\
\text { inusuales }\end{array}$ & $\begin{array}{c}\text { La batería está } \\
\text { descargada }\end{array}$ & Correcto \\
\hline 2 & $\begin{array}{l}\text { El aire } \\
\text { acondicionado } \\
\text { no funciona, no } \\
\text { refrigera }\end{array}$ & $\begin{array}{l}\text { Falta de gas en } \\
\text { el sistema }\end{array}$ & Correcto \\
\hline 3 & $\begin{array}{l}\text { Han } \\
\text { transcurrido } 6 \\
\text { meses desde la } \\
\text { última revisión }\end{array}$ & $\begin{array}{l}\text { Debe revisar el } \\
\text { líquido de } \\
\text { frenos }\end{array}$ & Correcto \\
\hline 4 & Luces débiles & $\begin{array}{c}\text { Los faros } \\
\text { tienen una capa } \\
\text { que reduce la } \\
\text { capacidad de } \\
\text { iluminación } \\
\end{array}$ & Correcto \\
\hline 5 & \begin{tabular}{|l} 
El aire \\
acondicionado \\
tiene olores \\
inusuales \\
\end{tabular} & $\begin{array}{l}\text { Realizar una } \\
\text { revisión en el } \\
\text { filtro }\end{array}$ & Correcto \\
\hline 6 & $\begin{array}{l}\text { El conductor } \\
\text { cree tener } \\
\text { problemas con } \\
\text { el radiador } \\
\end{array}$ & $\begin{array}{l}\text { El sistema no } \\
\text { encontró fallas }\end{array}$ & Correcto \\
\hline 7 & $\begin{array}{l}\text { No funciona el } \\
\text { acelerador del } \\
\text { auto } \\
\end{array}$ & $\begin{array}{l}\text { Obstrucción del } \\
\text { filtro de } \\
\text { gasolina } \\
\end{array}$ & Correcto \\
\hline 8 & $\begin{array}{l}\text { Problemas para } \\
\text { arrancar el auto }\end{array}$ & $\begin{array}{c}\text { Batería } \\
\text { desgastada o } \\
\text { quemada }\end{array}$ & Correcto \\
\hline
\end{tabular}

\section{Conclusiones}

En definitiva, los avances tecnológicos han sido siempre cada vez mayores a lo largo de la historia de las ciencias computacionales. La Inteligencia Artificial ha podido contribuir a la sociedad con muchas herramientas para apoyar al ser humano en su vida cotidiana, automatizando diversas actividades comunes. Un ejemplo de esto han sido los sistemas expertos.

En este proyecto se planteado una aplicación de software, específicamente un sistema experto que ayude a diagnosticar las fallas mecánicas y eléctricas más comunes en los automóviles. Este sistema está dirigido a los usuarios que no tienen conocimientos básicos en el área de mecánica de automóviles.
El objetivo principal es que los usuarios puedan desde sus hogares hacer un diagnóstico previo de un problema en su vehículo, y a partir de allí, tomar decisiones. Este diagnóstico previo puede ayudar a ahorrar tiempo, y dinero en aquellos casos donde la avería puede ser sencilla de resolver, sin necesidad de ir a un taller especializado.

Este es un primer prototipo construido, que requiere mayor investigación y pruebas para mejorar la fiabilidad del sistema.

\section{REFERENCIAS}

[1] D. Pachamora Pinedo, "Sistemas expertos: una opción de solución confiable," Universidad Peruana Unión, diciembre, 2019. [En línea]. Disponible en: http://repositorio.upeu.edu.pe/handle/UPEU/2318. [Accedido: 13-feb-2021.]

[2] R. Fuentes Covarrubias y A. Fuentes Covarrubias, "Desarrollo de un sistema experto para el diagnóstico de fallas automotrices," TE Rev. Iberoam. Tecnol. en Educ. y Educ. en Tecnol., no. 11, pp. 83-91, 2013.

[3] L. Andrés, M. Ortega, O. Fausto, and R. Galaviz, "Análisis y clasificación de sonidos de motores de carros, para diagnosticar fallas, con ayuda de redes neuronales," 2009. [En línea]. Disponible en: http://ingenieria.uatx.mx/http://www.itapizaco.edu.mx/ [Accedido: 31-mar-2021.

[4] C. C. Cepeda Landin y J. A. Rivera Caravajo, "Diagnóstico de fallas de un motor de encendido provocado corsa Evolution 1.41 mediante el análisis de las señales de los sensores MAP, TPS, VSS, utilizando herramientas de aprendizaje y clasificación," tesis de grado, Universidad Politécnica Salesiana, Cuenca, 2017. [En línea]. Disponible en: http://dspace.ups.edu.ec/handle/123456789/14735 [Accedido: 31-mar-2021].

[5] L. Copacondori, "Desarrollo de un sistema de diagnóstico de fallas en la dirección asistida eléctrica de automóviles," tesis de maestría, Escuela de Posgrado, Pontificia Universidad Católica del Perú, 2017.

[6] E. A. Torres Vargas, "Sistema de diagnóstico automotriz mediante el análisis de emisiones contaminantes con la aplicación de redes neuronales para la detección de fallas," tesis de grado, Universidad Politécnica Salesiana, Cuenca, 2017.

[7] V. M. Menacho López, "Modelo computacional basado en sistemas expertos para la gestión de mantenimiento de maquinarias de movimiento de tierras de la ciudad de Huaraz 2016," tesis doctoral, Escuela de Postgrado, Universidad Nacional Santiago Antúnez De Mayolo, Perú, 2017.

[8] R. Lahoz-Beltrá, Bioinformática: Simulación, vida artificial e inteligencia artificial. Madrid, España: Ediciones Díaz de Santos, 2004, 2004.

[9] S. Badaró, L. Javier Ibañez, and M. J. Agüero, "Sistemas expertos: fundamentos, metodologías y aplicaciones," 2013. [En línea]. Disponible en: https://dialnet.unirioja.es/servlet/articulo? codigo $=4843871$ [Accedido: 13-feb-2021].

[10] E. Ariel Sierra y A. Y. Hossian Ramón García-Martínez, 
Pinzón (et al): Propuesta de prototipo de un sistema experto para el diagnóstico mecánico y eléctrico de automóviles

"Sistemas expertos que recomiendan estrategias de instrucción. un modelo para su desarrollo," Revista Latinoamericana De Tecnología Educativa, ISSN-e 1695-288X, Vol 1, No 1, 2002 , págs 26-40. 2002. Disponible en: from: https://dialnet.unirioja.es/servlet/articulo?codigo=1252608\&in fo=resumen\&idioma $=$ SPA

[11] C. Spenser, "Drawing on your knowledge with VisiRule," in IEEE Potentials, vol. 26, no. 1, pp. 20-25, enero-febrero, 2007, doi: 10.1109/MP.2007.343036.

[12] K. Santiago Santiago, "Sistema basado en conocimiento para identificar problemas complejos y proponer estrategias que mejoren el funcionamiento del proceso de diseño en la industria," tesis doctoral, Universidad Autónoma Metropolitana Azcapotzalco, 2013.

[13] Instution for Human and Machine Cognition. "CmapTools | Cmap.” Disponible en: https://cmap.ihmc.us/cmaptools/ 